\title{
A Six-Year Retrospective Follow-up of 502 Patients with Laparoscopic Cholelithotomy for Gallstone Recurrence: Success or Prospect
}

Garba Seydou Aliou ( $\sim$ leroik@163.com )

Tongji University Affiliated Shanghai East Hospital

Gang Zhao

Shanghai East Hospital

An Hua Huang

Shanghai East Hospital

An An Xu

Shanghai East Hospital

Jing Li Cai

Shanghai East Hospital

Yu Long Yang

Shanghai East Hospital

Boureima Ali Nafissatou

Hopital National de Niamey

Hai Hu

Shanghai East Hospital

\section{Research article}

Keywords: gallstone; recurrence rate; laparoscopic cholelithotomy; risk factors; tauro-ursodeoxycholic acid, incision site.

Posted Date: July 3rd, 2019

DOl: https://doi.org/10.21203/rs.2.11013/v1

License: (c) (i) This work is licensed under a Creative Commons Attribution 4.0 International License. Read Full License 


\section{Abstract}

Background This research was designed to explore the risk factors for gallstone recurrence after laparoscopic cholelithotomy. Methods A total of 502 patients who were diagnosed with gallstones using ultrasonography underwent laparoscopic cholelithotomy between January 2011 and December 2017 at the Shanghai-East Affiliated Hospital of Tongji University. Results Our retrospective study revealed that the gallstone recurrence rate of patients taking tauro-ursodeoxycholic acid (TUDCA) was significantly lower $(P<0.05)$ than that of patients not taking TUDCA. The recurrence rate of gallstones in patients with an incision at the fundus of the gallbladder was significantly lower than that of the patients with an incision on the body of the gallbladder. The risk of recurrence in patients with gallstones combined with polyps was significantly higher than that in patients without polyps; the risk of recurrence of gallstones in patients with gallbladder contraction function $<50 \%$ was significantly higher than that in patients with gallbladder contraction function $\geq 50 \%$. Additionally, the prognosis of patients without gallbladder adhesions to the peritoneum was better than that of patients with adhesions. Conclusion During the 6year follow-up period of this study, the recurrence rate of gallstones after laparoscopic gallbladderpreserving cholelithotomy (LGPC) was $22.91 \%$. Factors related to gallstone recurrence were use of TUDCA, location of the incision, presence of gallstones combined with polyps, gallbladder contraction function and presence of gallbladder adhesions to the peritoneum. The main cause of gallstone recurrence needs further investigation, and laparoscopic cholelithotomy remains promising for treatment of gallstone recurrence but requires thorough follow-up.

\section{Background}

The gallbladder is a pear-shaped elastic sac located under the ribcage and below the liver. This organ concentrates and stores bile ${ }^{1}$. With the advent of minimally invasive surgery and increasing clinical application of laparoscopy, laparoscopic cholelithotripsy has been widely used to remove gallstones. Studies have shown that laparoscopic cholelithotripsy has many advantages over traditional open cholelithotripsy, such as less trauma, greater safety, shorter hospital stay, fewer complications, and faster recovery, but it has a particular risk of gallstone recurrence ${ }^{2}$. Other researchers have found that the recurrence rate of gallstones after minimally invasive cholecystolithotomy is not high, although residual gallstones cause most of the recurrence of gallstones following the operation. Thus, as long as the incidence of residual gallstones is reduced as much as possible, the recurrence rate of cholecystolithiasis can be minimized after the operation, and the gallbladder can also be preserved ${ }^{3}$. Pathogenic studies have pointed out that the recurrence of gallstones is the most important complication of gallbladderconserving surgery, and the recurrence rate of gallstones after gallstone-conserving surgery is entirely variable ${ }^{4}$. Therefore, the recurrence rate of gallstones after choledochotomy is controversial. It was believed that the factors influencing the recurrence of calculi in different studies vary, which may be an important reason for the various findings. Some physicians believe that the high recurrence rate of gallstones after gallstone-conserving surgery is mainly due to residual gallstones, mucosal injury and bleeding during gallstone removal ${ }^{5}$. 
Additionally, family history, body mass index (BMI) and diabetes are independent risk factors for gallstone recurrence after surgery for cholecystolithiasis with gallbladder preservation. Furthermore, gallbladder function, gallbladder wall thickness, blood triglyceride level, and use of ursodeoxycholic acid after surgery for cholecystolithiasis with gallbladder preservation are closely associated with gallstone recurrence after the procedure ${ }^{6}$. Therefore, to decrease the recurrence rate of gallstones after cholecystectomy, it is necessary to consider the factors affecting the recurrence of gallstones with a comprehensive analysis including the following: a comprehensive pre-operative assessment of gallbladder function where the indication of cholecystectomy should be strict, an intra-operative assurance of a high stone clearance rate, a post-operative reduction of triglyceride level by diet and exercise and a post-operative application of ursodeoxycholic acid/cholinergic acid.

At present, there is no medical-based evidence for gallstone-conserving lithotripsy in a prospective, largescale, multi-user randomized controlled study. Currently, the "gold standard" for the treatment of gallstones is still laparoscopic cholecystectomy, while a gallstone-preserving operation has not been included in the routine diagnosis and treatment of gallstone patients. Consequently, the gallstonepreserving operation for gallstone patients requires careful attention, and it inspires us to further explore the risk factors for recurrence of gallstones after laparoscopic cholecystolithotripsy. This study collectively selected the medical records of patients treated with laparoscopic, gallbladder-preserving cholelithotomy and analysed the gallstone recurrence rates and associated risk factors.

\section{Methods}

Patients' follow-up data were collected after laparoscopic cholelithotomy between January 2011 and December 2017 at the Shanghai-East Affiliated Hospital of Tongji University, Tongji Medical School, Shanghai City, China. The study was derived from 750 patients diagnosed with gallstones who had been systematically entered into the cholecystolithiasis registry of the General Surgery Department's Gallbladder Disease Centre and underwent laparoscopic gallbladder-preserving cholelithotomy. Follow-up was carried out at regular outpatient visits $(n=293)$ or by telephone contact $(n=209)$. A total of 502 patients $(66.93 \%)$ responded to the follow-up and hence participated in the study. (Fig. 1. a)

Patients' age, sex, history of gallstone disease, history of operation, data on chronic diseases (particularly hypertension, diabetes, hepatopathy), and cholelithotomy information, such as surgical approach, incision location, adhesion, gallbladder bleeding, gallbladder wall thickness, gallbladder contraction function, fatty liver, stone size-composition-morphology and number, presence of gallstone with adenopathy, hepatic cyst, TUDCA administration, hyperlipidaemia, and BMI were collected with a questionnaire.

\section{Statistical analysis}

Data were analysed by SPSS software 20.0 ( IBM SPSS statistics for Windows ). Univariate analyses with Cox proportional hazards models were performed to assess the association between each variable 
and outcome (Table 1). Statistically significant variables with a P-value $<0.05$ retained in the multivariate model along with age and sex were considered relevant. Multivariate analyses were performed using a backward-conditional selection procedure. The Kaplan-Meier method was used to estimate survival probabilities for the combined endpoint, which was defined as either the recurrence of gallstone or no recurrence of gallstones in the 6-year study period.

\section{Results}

Data from a total of 750 patients who underwent laparoscopic cholelithotomy, specifically gallbladderpreserving cholelithotomy, were analysed. After six years of follow-up, 502 patients participated in this study, among which 115 patients had gallstone recurrence, representing approximately $22.91 \%$ of the overall gallstone recurrence rate within the six year study period. In addition, we investigated the risk factors associated with gallstone recurrence rate, and we found five major factors associated with gallstone recurrence in patients after laparoscopic cholelithotomy: administration of TUDCA, gallstones combined with polyps, gallbladder contraction function, cumulative recurrence, and incision location.

\section{TUDCA-treated patients have greater relapse risk}

A total of 189 patients (37.6\%) did not use TUDCA. We found that 129 patients (25.6\%) had no recurrence, and 60 patients (12\%) had gallstone recurrence. In addition, we found that $313(62.4 \%)$ patients took TUDCA, whereas 258 patients $(51.4 \%)$ had no gallstone recurrence, but 55 patients $(11 \%)$ had gallstone recurrence. Overall, our data showed that the risk of relapse in patients treated with TUDCA was 0.620 times greater than that in patients not taking TUDCA (Log-Rank $=8.672 ; p=0.003$ ) (Fig. 1. b).

\section{The risk of gallstone recurrence in patients with polyps}

Our data revealed 468 patients (93.2\%) without reported polyps, 367 patients $(73.1 \%)$ had no gallstone recurrence, but 101 patients $(20.1 \%)$ had gallstone recurrence. There were 34 patients $(6.8 \%)$ with polyps, 20 patients $(4 \%)$ had no gallstone recurrence, and 14 patients $(2.8 \%)$ had gallstone recurrence after laparoscopic cholelithotomy.

We found that patients with gallstones combined with polyps had a 2.508-fold higher risk of gallstone recurrence than patients without polyps (Log-Rank = 11.076; $p=0.001$ ) (Fig. 2. c).

\section{Gallbladder contraction function}

In our dataset, 114 patients $(22.7 \%)$ had gallbladder contraction function $<50 \%$. Seventy-seven patients (15.3\%) had no gallstone recurrence after laparoscopic cholelithotomy, but 37 patients $(7.4 \%)$ had gallstone recurrence. Moreover, we found that 388 patients $(77.3 \%)$ had gallbladder contraction function $\geq 50 \%$. A total of 310 patients $(61.8 \%)$ had no gallstone recurrence, while 78 patients $(15.5 \%)$ had gallstone recurrence. 
The risk of gallstone recurrence in patients with gallbladder contraction function $<50 \%$ was 0.544 times greater than that in patients with gallbladder contraction function $\geq 50 \%($ Log-Rank $=13.571 ; p=0.001)$ (Fig. 2. d).

\section{The cumulative recurrence curve}

A total of 375 patients $(74.7 \%)$ had gallbladder adhesion to the peritoneum; of these, 294 patients (58.6\%) had no gallstone recurrence, but 81 patients $(16.1 \%)$ had gallstone recurrence after laparoscopic cholelithotomy. On the other hand, we found that 127 (25.3\%) patients with a gallbladder had no adhesion to the peritoneum. There were 93 patients (18.5\%) with no gallstone recurrence, and 34 patients (6.8\%) with gallstone recurrences.

The cumulative recurrence curve was significantly different for patients with gallbladder and peritoneum adhesion versus patients without gallbladder adhesion to the peritoneum ( $\log$ Rank $=4.647, \mathrm{P}=0.031$ ) (Fig. 3. e).

\section{The incision location}

Furthermore, our dataset contained 20 patients (4\%) who had a gallbladder neck incision. Among them, 13 patients $(2.6 \%)$ had no gallstone recurrence, and 7 patients $(1.4 \%)$ had gallstone recurrence. However, the majority of cases, approximately 415 patients (82.7\%), had an incision on the gallbladder fundus; among these, 31 patients $(65.9 \%)$ had no gallstone recurrence, 84 patients $(16.7 \%)$ had gallstone recurrence. In addition, 67 patients (13.3\%) had an incision on the gallbladder body, 43 patients $(8.6 \%)$ had no gallstone recurrence, and 24 patients $(4.8 \%)$ had gallstone recurrence after laparoscopic cholelithotomy according to our dataset.

The risk of recurrence in patients with an incision at the fundus of the gallbladder was 0.497 times lower than that of patients with an incision made at the gallbladder body (95\% Cl: $0.314-0.789)$ (Fig. 3. f).

Overall, the univariate analysis of the data (Table 2) showed TUDCA $(P<0.05)$, incision location $(P<$ $0.05)$, gallbladder with peritoneum adhesion $(p=0.031)$, gallstones combined with polyps $(0.001)$ and gallbladder contraction function $(P<0.05)$ to be significant risk factors for gallstone recurrence. Multivariate analysis (Table 3) showed TUDCA (HR 6.432, CL 0.428-0.897, P = 0.011), incision location (1) (HR 8.818, CL 0.314-0.789, P = 0.003), stone combined with polyps (HR 10.098, CL 1.422-4.423, P = 0.001 ), and gallbladder contraction function (HR 9.019, CL 0.365-0.809, $P=0.003$ ) to be significant risk factors for gallstone recurrence.

\section{Discussion}

Cholecystolithiasis is a common and frequently occurring disease in the biliary tract system ${ }^{7}$. The incidence of cholelithiasis has gradually increased with the improvement of peoples' living standards ${ }^{8}$. At present, the prevalence of cholelithiasis has reached $10 \%$ in China, accounting for approximately $11.5 \%$ 
of hospitalized general surgery patients ${ }^{9}$. For gallstones with symptoms and complications, surgical treatment is the primary treatment, but whether gallstones are treated with gallbladder preservation approaches remains controversial ${ }^{10}$. Laparoscopic cholelithotripsy has the advantages of small incisions, less pain, quick recovery, less injury and ease of acceptance by patients ${ }^{11}$. However, after clinical follow-up, it was found that the operation has the disadvantage of gallstone recurrence ${ }^{12}$. Recently, with the broader application of laparoscopy, laparoscopic cholecystolithotripsy has been favoured by an increasing number of patients as well as clinicians ${ }^{13}$. It has many physiological advantages, such as minimal invasion and preservation gallbladder function. The whole gallbladder can be directly observed, and the suitability of the patient for cholecystolithotripsy can be determined by evaluating gallbladder function. Compared with traditional cholecystectomy, laparoscopic cholecystolithotomy can improve gallstone clearance rate ${ }^{14}$. However, clinical studies have found that laparoscopic cholecystectomy has a higher gallstone recurrence rate than cholecystectomy ${ }^{15}$. Gallstones are mainly cholesterol stones or of mixed composition, such as melanin, with complex causes. They are related to long-term consumption of a high fat diet, an increase of cholesterol content in the blood and the weakening of gallbladder contractility. It was found that any factor affecting the ratio of cholesterol to bile acids and phospholipids that causes cholestasis can lead to gallstone formation ${ }^{16}$.

The high recurrence rate of calculi after an operation in patients with a family history of calculi may be related to heredity and family eating habits. Genetic factors are important risk factors for gallstones ${ }^{17}$.

Post-operative patients with a high fat diet, high blood lipids and $\mathrm{BMI}>25$ are prone to gallstone formation due to cholesterol deposition in the gallbladder, imbalance of bile acid and high phospholipid concentration ${ }^{18}$.

Hypertensive patients often suffer from vascular endothelial injury, such as atherosclerosis with impaired balance of the endocrine system and reduced blood supply to the gallbladder, eventually resulting in the weakening of gallbladder contraction, cholestasis and gallstone formation.

A large number of stones or sediment will augment gallbladder inflammation, causing small residual gallstones to form stone centres and eventually gallstone recurrence ${ }^{19}$.

TUDCA is currently prescribed in the treatment of cholelithiasis, especially in patients with mild symptoms (i.e., no pancreatitis, no cholecystitis or no cholangitis) and patients with calculi $(<10 \mathrm{~mm})$ and cholesterol stones with normal gallbladder function ${ }^{20}$. Based on this study, the gallstone recurrence rate of the post-operative TUDCA treatment group is significantly lower than that of the non-drug treatment group, indicating that the administration of TUDCA can extend the post-operative prevention of recurrence.

Moreover, our findings showed that the gallstone recurrence rate of patients with surgical incisions located at the fundus of the gallbladder was significantly lower than that of patients with an incision situated in the body of the gallbladder. It has been reported that after choledocholithotomy, the elasticity 
of the wall of the common bile duct weakens, and the bile duct dilates due to chronic inflammation and fibrosis of the wall of the common bile duct ${ }^{21}$. Periampullary diverticulum squeezes the Oddi sphincter at the end of the common bile duct, resulting in mechanical obstruction or dyskinesia of the sphincter ${ }^{22}$. As a result, the incidence of cholestasis and bacterial infection in the bile duct increases.

Our study is consistent with previous findings ${ }^{23}$ that the incision location becomes a potential risk factor for recurrence of common bile duct stones. Therefore, a surgical incision located at the fundus reduces the pressure on the wall of the common bile duct. Additionally, the recurrence of non-peritoneum adhesion patients is better than that of peritoneum adhesion patients. The reason may be that peritoneum adhesion can affect the normal physiological function of the bile duct. Bile duct dysfunction can deteriorate the hydrostatic force of the bile, leading to slow bile flow or poor drainage. Thus, the solid components in bile are more likely to deposit and then lead to the recurrence of gallstones ${ }^{24}$.

Our study also shows that the recurrence risk of polyp patients is significantly higher than that of nonpolyp patients, which is consistent with the related research results indicating higher gallstone recurrence risk of polyp patients ${ }^{25}$. To the best of our knowledge, this is the first study on risk factors for gallstone recurrence, which include TUDCA prescription, incision location, number of gallstones, and peritoneum adhesion, based on a retrospective analysis.

\section{Conclusions}

Gallstone recurrence-associated factors were TUDCA, incision site, gallstones combined with polyps, gallbladder contraction function and gallbladder adhesions to the peritoneum. Adequate measures to avoid the latter risks will be promising for positive outcomes. However, to provide better prevention and treatment plans for the non-recurrence of common bile duct stones, the risk factors that affect the recurrence rate of gallstones in laparoscopic cholelithotomy with gallbladder preservation demand further inquiry by prospective, multi-centre cohort studies.

\section{Abbreviations}

TUDCA : Tauro-ursodeoxycholic acid

LGPC : Laparoscopic gallbladder-preserving cholelithotomy

BMI : Body mass index

\section{Declarations}

\section{Acknowledgements}

The authors acknowledge the staff and doctors of the Gallbladder Disease Centre affiliated with Shanghai-East Hospital of Tongji University for their kind assistance in this study. 


\section{Authors' contributions}

Garba Seydou Aliou designed the research and facilitated the data collection. Hua Huang performed the statistical analysis. Gang Zhao and An An Xu contributed to the follow-up and consent form application, and Boureima Ali Nafissatou and Yu Long Yang contributed to the manuscript revision. All authors contributed to the drafting and final writing of the manuscript. Hu Hai and all co-authors approved the article's submission for publication.

\section{Funding}

This work was supported by the Natural Science Foundation of China (81670579) and the National Natural Science Foundation of China under Grant number (91570577).

\section{Availability of data and materials}

The datasets supporting the conclusions of this article are included within the article.

\section{Conflict of interest}

The authors declare that they have no conflict of interest.

\section{Ethical statement}

This study was approved by the Shanghai Ethical Committee of Laboratory Animals and Human Studies, Tongji University branch, and consent was obtained from all patients prior to the study.

\section{Competing interests}

The authors report no proprietary or commercial interest in any product mentioned or concept discussed in this article.

\section{References}

1. Bustos BI, Perez-Palma E, Buch S, Azocar L, Riveras E, Ugarte GD, et al. Variants in ABCG8 and TRAF3 genes confer risk for gallstone disease in admixed Latinos with Mapuche Native American ancestry. Sci Rep. 2019;9(1):772.

2. Han T, Lv Y, Wang S, Hu T, Hong H, Fu Z. PPARgamma overexpression regulates cholesterol metabolism in human L02 hepatocytes. J Pharmacol Sci. 2019;139(1):1-8.

3. Zhang Y, Peng J, Li X, Liao M. Endoscopic-Laparoscopic Cholecystolithotomy in Treatment of Cholecystolithiasis Compared With Traditional Laparoscopic Cholecystectomy. Surg Laparosc Endosc Percutan Tech. 2016;26(5):377-80.

4. Han IW, Kwon OC, Oh MG, Choi YS, Lee SE. Effects of Rowachol on prevention of postcholecystectomy pain after laparoscopic cholecystectomy: prospective multicenter randomized 
controlled trial. HPB (Oxford). 2016;18(8):664-70.

5. Kim H, Han IW, Heo JS, Oh MG, Lim CY, Choi YS, et al. Postcholecystectomy syndrome: symptom clusters after laparoscopic cholecystectomy. Ann Surg Treat Res. 2018;95(3):135-40.

6. Zha Y, Chen XR, Luo D, Jin Y. The prevention of major bile duct injures in laparoscopic cholecystectomy: the experience with 13,000 patients in a single center. Surg Laparosc Endosc Percutan Tech. 2010;20(6):378-83.

7. Halldorsson MO, Hauptmann M, Snaebjornsson P, Haraldsdottir KH, Aspelund T, Gudmundsson EF, et al. The risk of developing a mismatch repair deficient colorectal cancer after undergoing cholecystectomy. Scand J Gastroenterol. 2018;53(8):972-5.

8. Atak I, Ozdil K, Yucel M, Caliskan M, Kilic A, Erdem H, et al. The effect of laparoscopic cholecystectomy on the development of alkaline reflux gastritis and intestinal metaplasia. Hepatogastroenterology. 2012;59(113):59-61.

9. Zha Y, Zhou ZZ, Chen XR, Gan P, Tan J. Gallbladder-preserving cholelithotomy in laparoscopic and flexible choledochoscopic era: a report of 316 cases. Surg Laparosc Endosc Percutan Tech. 2013;23(2):167-70.

10. Wang T, Chen T, Zou S, Lin N, Liang HY, Yan HT, et al. Ultrasound-guided double-tract percutaneous cholecystostomy combined with a choledochoscope for performing cholecystolithotomies in highrisk surgical patients. Surg Endosc. 2014;28(7):2236-42.

11. Liu D S, Guan F, Wang B, et al. Combined usage with intraperitoneal and incisional ropivacaine reduces pain severity after laparoscopic cholecystectomy. International Journal of Clinical \& Experimental Medicine. 2015, 8(12):22460.

12. Liu B, Du B, Pan Y. Video of the Month: Transrectal Gallbladder-Preserving Cholecystolithotomy via Pure Natural Orifice Transluminal Endoscopic Surgery: First Time in Humans. Am J Gastroenterol. 2015;110(12):1655.

13. Ye L, Liu J, Tang Y, Yan J, Tao K, Wan C, et al. Endoscopic minimal invasive cholecystolithotomy vs laparoscopic cholecystectomy in treatment of cholecystolithiasis in China: a meta-analysis. Int $\mathrm{J}$ Surg. 2015;13:227-38.

14. Gilloteaux J, Ott D W, Oldham-Ott C K. The Gallbladder of the Electric Ray Torpedo Marmorata Risso Displays Excrescent Cholecystocytes with Merocrine and Apocrine-Like Secretions. Anatomical Record, 2013, 296(1):79-95..

15. Li QF, Xu X, Ge X. Gallstone recurrence after minimally-invasive cholecystolithotomy with gallbladder reservation: a follow-up of 720 cases. Eur Rev Med Pharmacol Sci. 2015;19(8):1403-6.

16. Wang T, Luo H, Yan HT, Zhang GH, Liu WH, Tang LJ. Risk factors for gallbladder contractility after cholecystolithotomy in elderly high-risk surgical patients. Clin Interv Aging. 2017;12:129-36.

17. Lin X, Cai J, Wang J, Chen C, He G, Han Y. Minimally Invasive Cholecystolithotomy to Treat Cholecystolithiasis in Children: A Single-center Experience With 23 Cases. Surg Laparosc Endosc Percutan Tech. 2017;27(5):e108-e10. 
18. Masuda Y, Mizuguchi Y, Kanda T, Furuki H, Mamada Y, Taniai N, et al. Successful treatment of limy bile syndrome extending to the common bile duct by laparoscopic cholecystectomy and common bile duct exploration: A case report and literature review. Asian J Endosc Surg. 2017;10(1):59-62.

19. Musleh MG, Burnett $H$, Rajashanker B, Ammori BJ. Laparoscopic double cholecystectomy for duplicated gallbladder: A case report. Int J Surg Case Rep. 2017;41:502-4.

20. Gupta R, Verma P, Yadav J, Singh AK, Bhat H, Singh SK. Laparoscopic Cholecystectomy for Gallbladder Duplication. J Gastrointest Surg. 2018.

21. Talseth A, Ness-Jensen E, Edna TH, Hveem K. Risk factors for requiring cholecystectomy for gallstone disease in a prospective population-based cohort study. Br J Surg. 2016;103(10):1350-7.

22. Comajuncosas J, Hermoso J, Gris P, Jimeno J, Orbeal R, Vallverdu H, et al. Risk factors for umbilical trocar site incisional hernia in laparoscopic cholecystectomy: a prospective 3-year follow-up study. Am J Surg. 2014;207(1):1-6.

23. Fukuba, N., Ishihara, S., Sonoyama, H., Yamashita, N., Aimi, M., \& Mishima, Y., et al. (2017). Proton pump inhibitor is a risk factor for recurrence of common bile duct stones after endoscopic sphincterotomy - propensity score matching analysis. Endoscopy International Open, 05(04), E291E296.

24. Yajima H, Kanai H, Son K, Yoshida K, Yanaga K. Reasons and risk factors for intraoperative conversion from laparoscopic to open cholecystectomy. Surg Today. 2014;44(1):80-3.

25. Hui, W. J., Park, S. J., Hong, S. P., Cheon, J. H., Kim, W. H., \& Kim, T. I. (2015). Risk factors for recurrent high-risk polyps after the removal of high-risk polyps at initial colonoscopy. Yonsei Medical Journal, 56(6), 1559-1565.

\section{Tables}

Table 1: Patient characteristics 


\begin{tabular}{|c|c|}
\hline Total & $\mathrm{n}(\%)$ \\
\hline \multicolumn{2}{|l|}{ Sex } \\
\hline Male & $184(36.7)$ \\
\hline Female & 318(63.3) \\
\hline \multicolumn{2}{|l|}{ Age } \\
\hline$<60$ & $434(86.5)$ \\
\hline$\geq 60$ & 68(13.5) \\
\hline \multicolumn{2}{|l|}{ Hypertension } \\
\hline No & $436(86.9)$ \\
\hline Yes & 66(13.1) \\
\hline \multicolumn{2}{|l|}{ Diabetes } \\
\hline No & $486(96.8)$ \\
\hline Yes & $16(3.2)$ \\
\hline \multicolumn{2}{|l|}{ TUDCA } \\
\hline No & 189(37.6) \\
\hline Yes & $313(62.4)$ \\
\hline \multicolumn{2}{|l|}{ Location of the incision } \\
\hline Neck & $20(4.0)$ \\
\hline Fundus & $415(82.7)$ \\
\hline Body & 67(13.3) \\
\hline \multicolumn{2}{|c|}{ Adhesion with peritoneum } \\
\hline No & $375(74.7)$ \\
\hline Yes & 127(25.3) \\
\hline \multicolumn{2}{|l|}{ Number of stones } \\
\hline Single stone & $251(50)$ \\
\hline More than one stone & $251(50)$ \\
\hline \multicolumn{2}{|c|}{ Stone combined with polyps } \\
\hline No & 468(93.2) \\
\hline Yes & $34(6.8)$ \\
\hline \multicolumn{2}{|l|}{ Size of the stone $(\mathrm{mm})$} \\
\hline$<2 \mathrm{~cm}$ & $354(70.5)$ \\
\hline$\geq 2 \mathrm{~cm}$ & 148(29.5) \\
\hline \multicolumn{2}{|c|}{ Gallbladder wall thickness (mm) } \\
\hline$\leq 3$ & 451(89.8) \\
\hline$>3$ & $51(10.2)$ \\
\hline \multicolumn{2}{|c|}{ Gallbladder contraction function } \\
\hline$<50 \%$ & $114(22.7)$ \\
\hline$\geq 50 \%$ & 388(77.3) \\
\hline \multicolumn{2}{|l|}{ BMI } \\
\hline$<24$ & $270(53.8)$ \\
\hline$\geq 24$ & $232(46.2)$ \\
\hline
\end{tabular}

Table 2 : Univariate Analyses 


\begin{tabular}{lrr}
\hline Group & Chi-square & P value \\
\hline Sex & 1.474 & 0.225 \\
\hline Age & 1.606 & 0.205 \\
\hline Onset of gallstone diseases & 2.633 & 0.621 \\
\hline History of operation & 1.054 & 0.305 \\
\hline Hypertension & 1.381 & 0.240 \\
\hline diabetes & 0.480 & 0.488 \\
\hline Liver disease & 0.212 & 0.899 \\
\hline Surgical approach & 2.190 & 0.139 \\
\hline TUDCA & 8.672 & 0.003 \\
\hline Stone comparison & 0.101 & 0.751 \\
\hline Stone structure & 0.874 & 0.646 \\
\hline Stone composition & 0.964 & 0.326 \\
\hline Location of the incision & 16.185 & $\mathbf{0 . 0 0 0}$ \\
\hline Adhesions & 4.647 & $\mathbf{0 . 0 3 1}$ \\
\hline Stone number & 0.079 & 0.779 \\
\hline Stone combined with polyps & 11.076 & 0.001 \\
\hline Location of the incision haematoma & 0.240 & 0.624 \\
\hline Gallbladder bleeding & 0.623 & 0.430 \\
\hline Size of stone & 0.021 & 0.886 \\
\hline Gallbladder wall & 4.935 & 0.177 \\
\hline Gallbladder wall thickness & 1.189 & 0.275 \\
\hline Gallbladder contraction function & 13.571 & $\mathbf{0 . 0 0 0}$ \\
\hline Fatty liver & 0.010 & 0.919 \\
\hline Stone with adenopathy & 0.463 & 0.496 \\
\hline Hepatic cyst & 0.109 & 0.742 \\
\hline Hyperlipidaemia & 0.179 & 0.672 \\
\hline BMI & 0.026 & 0.872 \\
\hline
\end{tabular}

Table 2 Meaningful indices of univariate analyses $(P<0.05)$ showed TUDCA $(p=003)$, location incision $(P<$ $0.05)$, gallbladder with peritoneum adhesion $(\mathrm{p}=0.031)$, gallstone combined with polyps $(\mathrm{p}=0.001)$ and gallbladder contraction function $(\mathrm{P}<0.05)$ to be significant factors for gallstone recurrence.

Table 3: Multivariate COX Regression Analysis

\begin{tabular}{lcccccccc}
\hline \multicolumn{1}{c}{ Variables } & B & SE & wald & df & Sig. & Exp(B) & \multicolumn{2}{c}{$\begin{array}{c}\text { E5.0\% Exp(B) CI } \\
\text { lower }\end{array}$} \\
& & & & & & & upper \\
\hline TUDCA & -0.479 & 0.189 & 6.432 & 1 & 0.011 & 0.620 & 0.428 & 0.897 \\
\hline Location of the incision & & & 10.634 & 2 & 0.009 & & & \\
\hline Location of the incision (1) & -0.698 & 0.235 & 8.818 & 1 & 0.003 & 0.497 & 0.314 & 0.789 \\
\hline Location of the incision (2) & -0.009 & 0.437 & 0.000 & 1 & 0.983 & 0.991 & 0.421 & 2.332 \\
\hline Stone combined with polyps & 0.920 & 0.289 & 10.098 & 1 & 0.001 & 2.508 & 1.422 & 4.423 \\
\hline Gallbladder contraction function & -0.609 & 0.203 & 9.019 & 1 & 0.003 & 0.544 & 0.365 & 0.809 \\
\hline
\end{tabular}

Table 3 COX regression analysis showed that Chi-square was 41.783, $\mathrm{P}<0.05$, indicating that the overall model had statistical significance. The final screened model included TUDCA; location of the incision; stone combined with polyps and gallbladder contraction function and was described as follows: TUDCA (HR 6.432, CL 0.4280.897, $\mathrm{P}=0.011$ ), location incision (1) (HR 8.818, CL 0.314-0.789, $\mathrm{P}=0.003$ ), stone combined with polyps (HR 
10.098, CL 1.422-4.423, $\mathrm{P}=0.001$ ) and gallbladder contraction function (HR 9.019, CL 0.365-0.809, $\mathrm{P}=0.003$ ) to be significant factors for gallstone recurrence

\section{Figures}

a

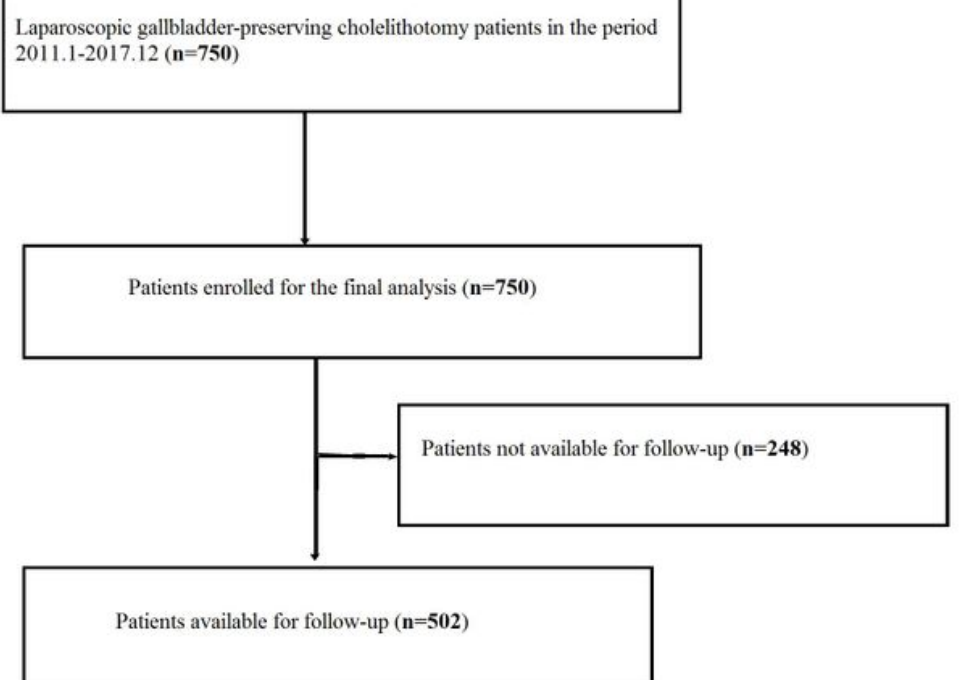

b

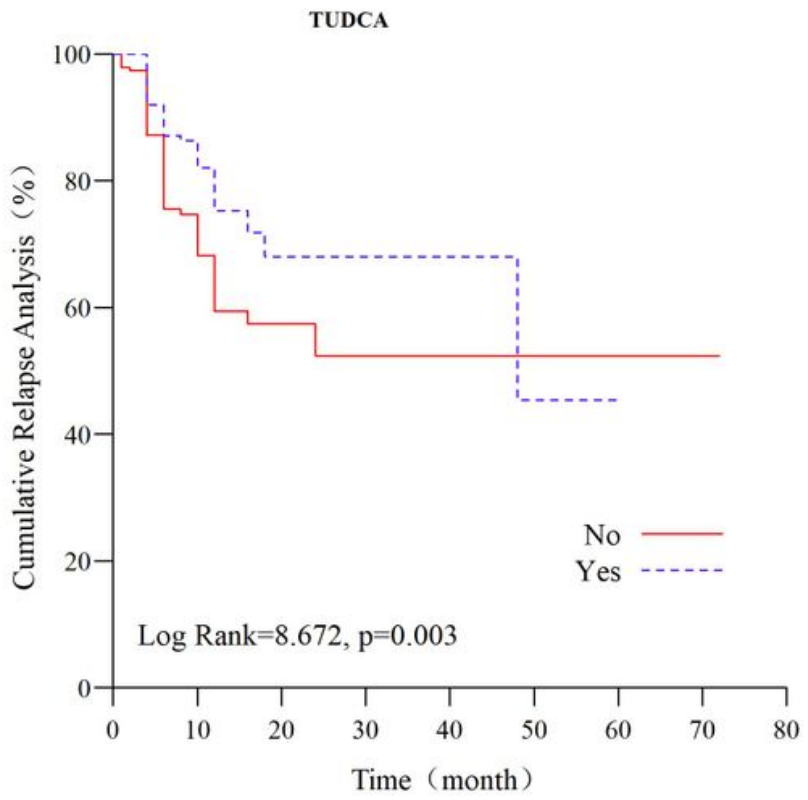

\section{Figure 1}

Fig. 1. a Flow diagram for the selection of laparoscopic gallbladder-preserving cholelithotomy patients. Fig. 1. b Use of TUDCA: the risk of relapse in TUDCA-treated patients is 0.620 times that in patients not taking TUDCA (Log-Rank = 8.672; $\mathrm{p}=0.003)$. 
c

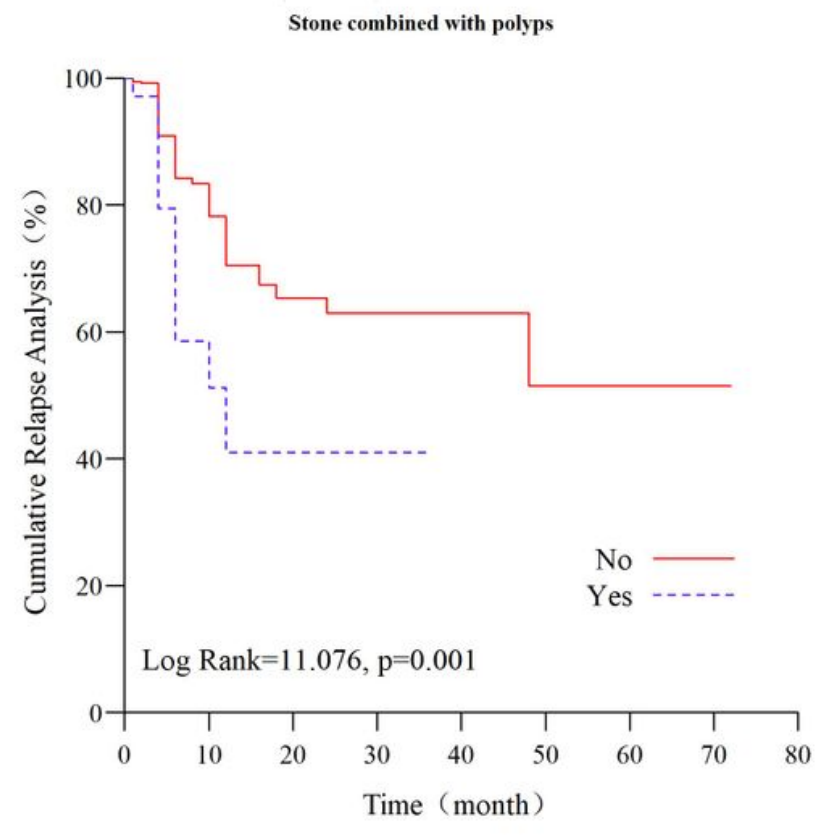

d

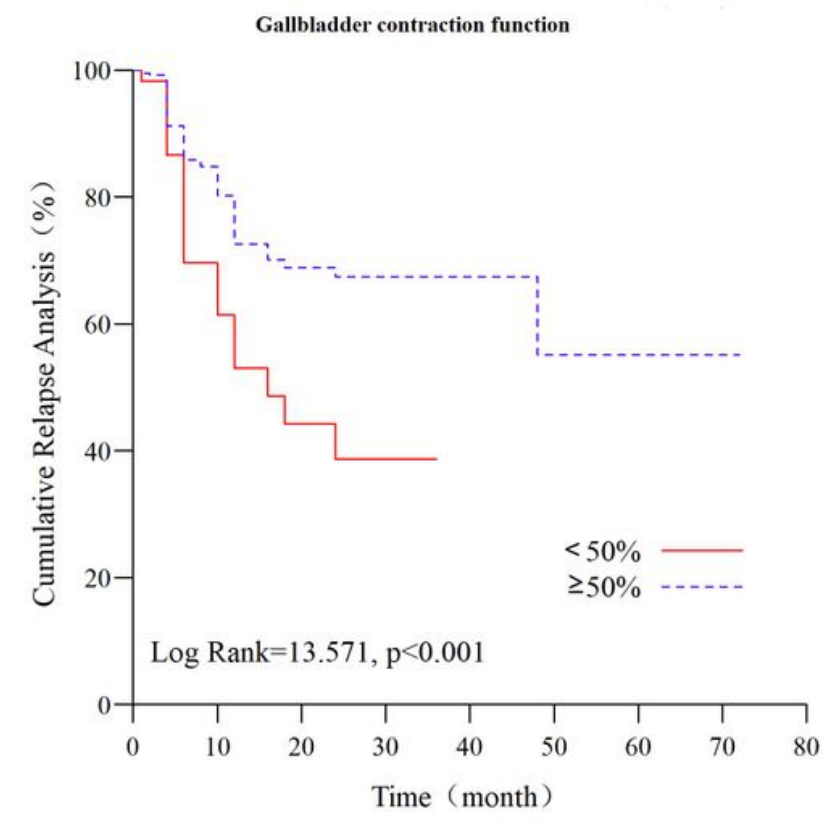

\section{Figure 2}

Fig. 2. c Gallstone combined with polyps: the risk of gallstone recurrence in patients with polyps was 2.508 times that in patients without polyps (Log-Rank $=11.076 ; p=0.001$ ). Fig. 2 . $d$ Gallbladder contraction function: the risk of gallstone recurrence in patients with gallbladder lock function $<50 \%$ was 0.544 times that in patients with gallbladder lock function $\geq 50 \%$ (Log-Rank $=13.571 ; p=0.001)$.

$\mathrm{e}$

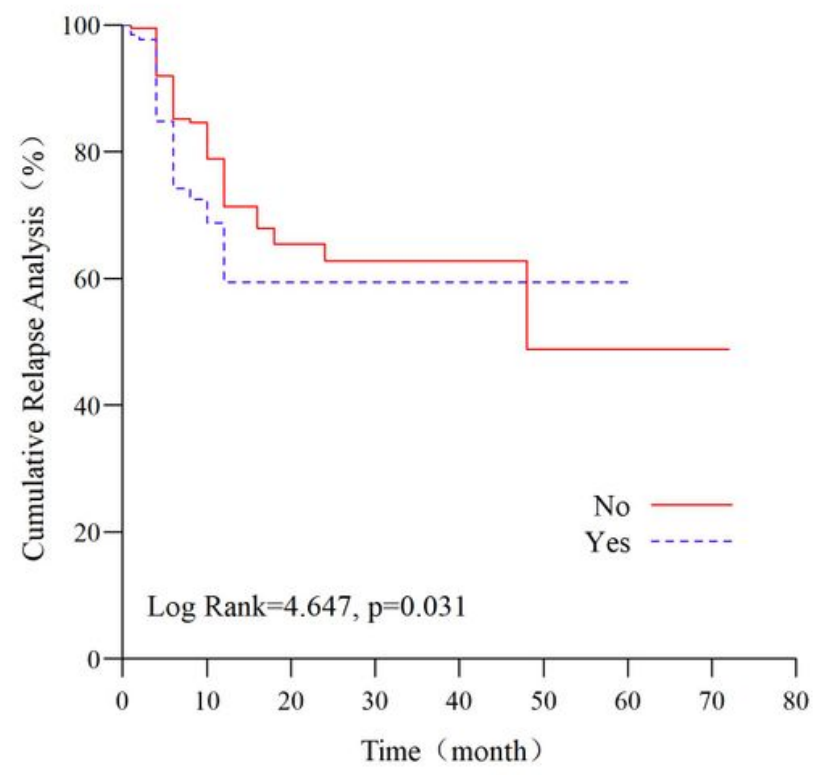

$\mathrm{f}$

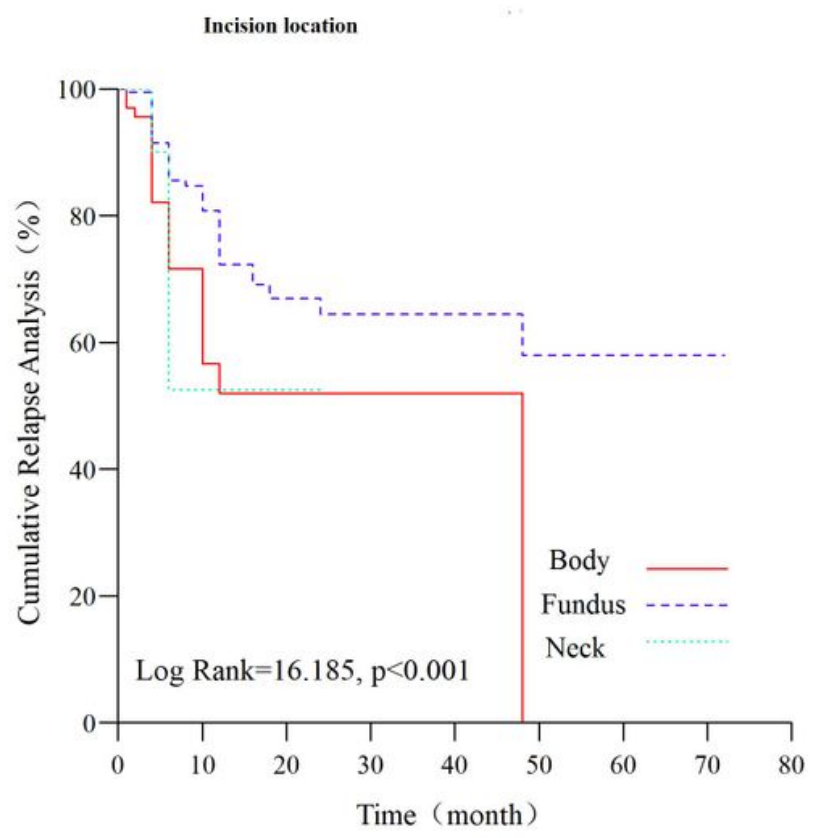

Figure 3 
Fig. 3. e The cumulative recurrence curve was significantly different in patients with gallbladder and peritoneum adhesion and patients without gallbladder adhesions to the peritoneum (Log Rank $=4.647 \mathrm{P}$ $=0.031)$. Fig. 3. $\mathrm{f}$ The incision location: the risk of gallstone recurrence in patients with an incision at the fundus of the gallbladder was 0.497 times lower than that in patients with an incision made at the gallbladder body (95\% Cl: 0.314-0.789). 